\title{
Effectiveness of a low-threshold physical activity intervention in residential aged care - results of a randomized controlled trial
}

This article was published in the following Dove Press journal:

Clinical Interventions in Aging

21 May 2015

Number of times this article has been viewed

\author{
Martin Cichocki \\ Viktoria Quehenberger ${ }^{\prime}$ \\ Michael Zeiler' \\ Tanja Adamcik' \\ Matthias Manousek' \\ Tanja Stamm² \\ Karl Krajic'
}

'Ludwig Boltzmann Institute Health Promotion Research, ${ }^{2}$ Medical

University of Vienna \& University of Applied Sciences FH Campus, Wien,

Vienna, Austria
Correspondence: Martin Cichocki Ludwig Boltzmann Institute Health Promotion Research, Untere Donaustrasse 47/3, 1020 Vienna, Austria Tel +43 I 212 I 493 10

Email cicho@gmx.net
Purpose: Research on effectiveness of low-threshold mobility interventions that are viable for users of residential aged care is scarce. Low-threshold is defined as keeping demands on organizations (staff skills, costs) and participants (health status, discipline) rather low. The study explored the effectiveness of a multi-faceted, low-threshold physical activity program in three residential aged-care facilities in Austria. Main goals were enhancement of mobility by conducting a multi-faceted training program to foster occupational performance and thus improve different aspects of health-related quality of life (QoL).

Participants and methods: The program consisted of a weekly session of 60 minutes over a period of 20 weeks. A standardized assessment of mobility status and health-related QoL was applied before and after the intervention. A total of 222 of 276 participants completed the randomized controlled trial study (intervention group $n=104$, control group $n=118$; average age 84 years, $88 \%$ female).

Results: Subjective health status (EuroQoL-5 dimensions: $P=0.001, d=0.36$ ) improved significantly in the intervention group, and there were also positive trends in occupational performance (Canadian Occupational Performance Measure). No clear effects were found concerning the functional and cognitive measures applied.

Conclusion: Thus, the low-threshold approach turned out to be effective primarily on subjective health-related QoL. This outcome could be a useful asset for organizations offering lowthreshold physical activity interventions.

Keywords: physical activity, intervention, residential aged care, effectiveness, aged

\section{Introduction}

The aging process is associated with loss of functional and physiological health. Disabilities - often related to chronic diseases - lead to limitations in activities of daily living (ADL) with an increased risk of becoming dependent on intensive nursing support in long-term care (LTC). ${ }^{1}$ Users of residential aged care (RAC) benefit from continuous professional nursing support in the management of their chronic conditions, but they are at higher risk of establishing a sedentary lifestyle as the RAC-organization is substituting for user's reduced capacities.

Even frail older persons can benefit from exercise programs in terms of positive training effects on physical fitness, functional, and ADL performance as well as on quality of life (QoL). ${ }^{2-4}$ Many RAC facilities provide exercise programs to enhance mobility of users, but systematic evaluation of the content of science-based exercise programs in RAC is primarily available for therapeutic and rehabilitation interventions. Most of the interventions within LTC include components aiming at muscle strengthening, flexibility, and balance, as well as reduction of specific mobility 
problems (eg, insecurity in walking and lacking endurance). They also aim at enhancing general daily living skills (eg, eating, dressing, and climbing stairs), and at increasing participation in social activities. Regarding scientific studies, there seems to be a trend to maximize health effects by increased frequency and intensity of training. The average intervention in the studies lasts 12 weeks with a modal number of three sessions per week. Most of the rehabilitation interventions are delivered by qualified health care professionals. $^{5}$

On the basis of several studies, a Dutch study group has developed an exercise protocol to maximize effects of physical activity (PA) programs for frail institutionalized older persons on physical fitness, functional, and ADL performance, and QoL. ${ }^{4}$ According to this recommendation, PA programs should combine resistance, balance, and functional training. The intensity of the training should be moderate to high and performed three times a week for at least 10 weeks.

For the implementation of a program following these recommendations, practical barriers can be encountered in the RAC setting that will be considered in this paper.

First, it is not clear if - or under what conditions - it is feasible for a pre-frail or frail population as institutionalized aged to attend a highly frequent and moderately intense exercise program. ${ }^{6,7}$ Lack of motivation and reduced capacities might hinder participation in highly intense training programs. A Canadian study stated that $98 \%$ of nursing homes provided some kind of exercise and activity program, but only $10 \%-15 \%$ of residents participated. ${ }^{8}$ Health plays an important role for participation in PA among institutionalized aged and highly-aged persons. The expectation of possible prevention of a decline in future health has a positive influence on participation while there are also negative influences, eg, the perceived level of pain. ${ }^{9}$

Second, the transferability of interventions for the aged to settings of RAC remains a challenge, ${ }^{10}$ especially for the organizations. With limited funding available for health promotion interventions in this sector, it is likely that intensive training programs will bear higher costs and will have less chance to be implemented by RAC providers on a routine basis.

Consequently, within the domain of PA programs in $\mathrm{RAC}$, there exists a need for programs, which are effective in promoting residents' health and simultaneously considers the needs of participants (eg, limited motivation and discipline, reduced functional health, frequent illness) as well as limited resources of organizations. Such low-threshold programs are more likely to become implemented, to remain viable in the routine of aged care services, and to have sustainable effects on residents' health.

\section{Patients and methods Primary outcome}

The main objective of the study is to explore effects of a lowthreshold, multi-faceted intervention on health-related QoL, physical functioning, and ADL of residents in RAC.

\section{Study setting}

The study was conducted as part of a larger health-promotion project in RAC in Vienna, involving three units of Austria's largest (semi-public) provider. ${ }^{11}$ The facilities offer a mix of assisted living and nursing care, combining different forms, ranging from apartments to nursing ward structures. There were no considerable differences in living conditions between the different locations and their residents. All participants were continuously living in RAC at the time of the study. Most residents choose to live in RAC due to existing problems in their ability to manage everyday routines or questionable abilities for safely living at home. In all facilities, gymnastic rooms or other adequate spaces for conducting a group exercise intervention were available. Since all participants lived in the RAC institutions, there was no need to consider aspects of transportation to follow the program.

\section{Study design}

The PA program was conducted between October 2011 and June 2012 as a registered randomized controlled trial (ISRCTN25536408) using standardized assessments to measure the health status of participants before and after the intervention. The ethics committee of the RAC provider approved the study protocol. Participants gave their written consent to be enrolled in the study.

The design of the intervention tried to optimize attractiveness for the central stakeholders by acknowledging interests, problems, and motives of residents, professional stakeholders (eg, experts in occupational therapy, local trainers with expertise in PA for users in RAC), and management. It was designed to allow for participation of a broad range of residents, including chronically ill, functionally impaired, and rather inactive elderly. For organizations, the low-threshold approach meant conducting an intervention that is close to established routines and available resources.

\section{Sample size}

Sample size calculations were performed by using $\mathrm{G}^{*}$ power 3.1.6 software. To detect a significant treatment effect operationalized by an interaction between group (intervention group [IG] versus [vs] control group [CG]) and time-point (baseline vs post-assessment), assuming a significance level of 0.05 , power of 0.80 , and a correlation of 0.50 between 
the time-points, a total of 100 participants per group was necessary. A dropout rate of $25 \%$ from baseline to postassessment was assumed, resulting in a target of about 125 participants to be initially included in the trial.

\section{Randomization method}

At the start of the study the three institutions comprised 878 inhabitants. Residents were approached following a randomly generated list by local project coordinators, who were part of the local RAC staff and therefore knew participants and their health status. Persons with no or mild physical and/or cognitive impairment were included; exclusion from participation was restricted to severe physical impairment (eg, being bedridden) or severe cognitive impairment. The selection was based on the coordinator's judgment supported by professional nurses from the ward and resulted in the exclusion of approximately 45 persons (5\%).

Following a randomly generated list, the coordinators individually and pro-actively invited residents and informed them about the personal relevance of the program. Many residents declined participation and so nearly all residents had to be approached to recruit one third of the users which was the number targeted for the study. After general agreement for participation in the study, residents were randomly allocated to either the IG $(n=139)$ or $\mathrm{CG}(\mathrm{n}=137)$. To randomly allocate the participants, a computerized random number generator was used based on alphabetical lists of the participants' names. Assessors were blinded to allocation; the list for distribution was safely kept and not passed on to assessors of the baseline and follow-up assessment. After completed allocation procedures, participants were informed whether they belonged to the IG or CG. The recruitment process and enrollment of participants is shown in Figure 1.

\section{Eligibility criteria}

All participants of the study were residents within the participating RAC facilities aged 65+. Participation in the study was advertised by staff members. Information about the meaning of mobility in the aged, the procedure, and aim of the study was given in announcements. All residents without severe physical and cognitive impairment could participate.

\section{Intervention design}

During the program period the IG took part in weekly PA exercise, consisting of 20 units for 60 minutes each week.

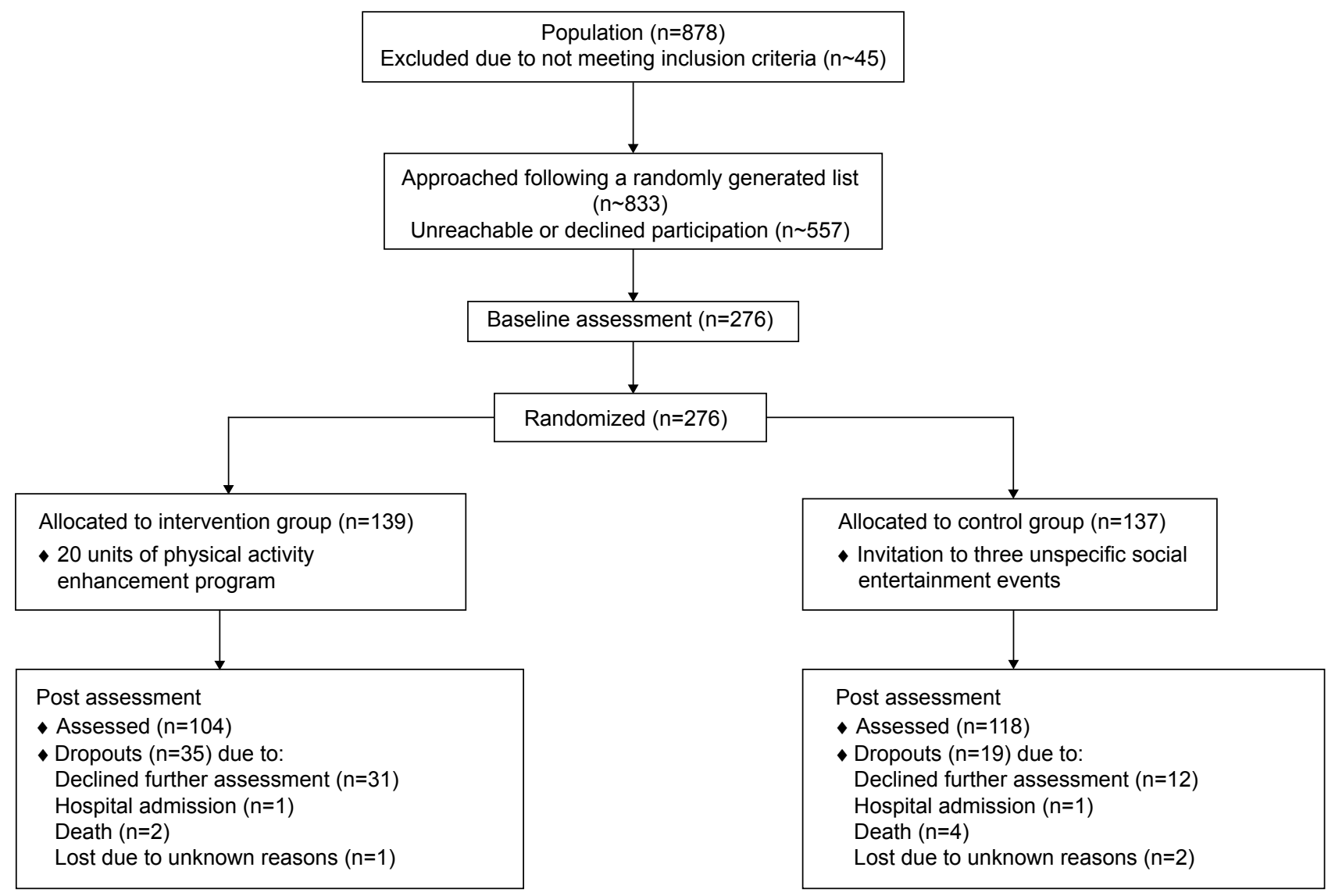

Figure I Recruitment process and enrollment of participants. 
The CG was invited to three social animation events with no specific focus on PA or associated skills.

Trainers and supervisors received an introductory training for the intervention program. Feasibility and viability were enhanced by fitting the program into the established framework of PA interventions in group settings.

The evidence-based curriculum of the PA program was designed by experts of occupational therapy at University of Vienna. ${ }^{12}$ The content of the units was selected based on an expert rating of existing evidence for interventions. ${ }^{13}$ The units were compiled following a complex understanding of health and mobility, including biological, psychological, and social dimensions. The units targeted a wide range of areas like coordination, balance, strength, endurance, sensorimotor perception, breathing, abilities, and skills for managing ADL, and interpersonal skills. An overview of the program is presented in Table 1.

The curriculum further specified didactical principles to be considered when implementing the intervention, providing the following guidance for the selection of exercises:

- exercises were expected to be of direct relevance for daily routines of aged persons;

- exercises were expected to involve dual-tasking;

- a central aim was to adapt exercises to individual needs and resources.

The principles of repetition and augmentation were to be considered when implementing exercises. Building upon a scientific basis, experts and practitioners for PA groups in aged care were consulted and involved in the development of the curriculum, thus to safeguard feasibility of the program taking the organization's and residents' resources into account. This led to adaptations toward a low-threshold design: staff, rooms, and equipment were planned to be primarily manageable with available organizational capacities. Trainers were primarily staff members of the RAC provider with experience in PA training for elderly persons; supervisors were physical or occupational therapists, who at certain points got practically involved in units with difficult tasks. Regarding the needs of residents the program was focused toward low intensity (eg, 20 weekly exercise units) and variety of content. A "home-exercise program" comprising ten exercises was added to promote participants' capacity to exercise independently.

\section{Assessment}

The assessment (before and after the intervention) was conducted by external experts in occupational therapy or physiotherapy using a standardized questionnaire. The assessment lasted 30-45 minutes and was conducted within the personal environment of the resident. Assessors were blinded to the participants' group allocation. After the baseline assessment no further exclusion of participants was necessary, because residents with severe limitations had been excluded beforehand.

The assessment included the following dimensions and instruments:

- Health-related QoL and subjective state of health were measured via "Euro Quality of Life-5 dimensions" (EQ-5D), extended by questions on mobility. ${ }^{14}$

- Pain status was assessed using a visual analog rating scale for perception of actual pain. ${ }^{15}$

- The Canadian Occupational Performance Measure (COPM) served as a gauge for performance of and satisfaction with everyday activity problems. ${ }^{16}$

- Functional tests included Timed Up and Go Test (TUAG), ${ }^{17-19}$ Chair Sit and Reach (CSAR) and Back Scratch (BS) Test, ${ }^{20}$ Lower Back Scratch and Neck Reach Test, measuring the range of motion of the upper extremities in relation to the cervical and lumbar spine. ${ }^{21}$

- Assessment of cognitive status included a shortened Mini-Mental State Examination (MMSE) to evaluate the participant's orientation regarding identity, time, space, and situation. ${ }^{22}$

- Quantification of and satisfaction with social contacts and mobility range of participants were measured with a set of items designed by the authors.

\section{Statistical analysis}

IBM SPSS Statistics 22.0 software was used for statistical analyses. Generalized linear mixed models for repeated measures (GLMMRM) were performed for metric outcome measures (eg, subjective health status, COPM performance, etc) to analyze differences between the intervention and CG. Data of functional outcomes (TUAG, CSAR, BS tests) were checked for extreme values and extreme pre-post changes ( $\pm>2$ standard deviations) prior to the analysis. Such values are very likely to be measurement errors or influenced by an actual medical condition and thus were omitted from the analysis. Implausible values exceeding the 95th percentile among community dwelling aged $80+$ years $^{23}$ were also excluded.

Primary analyses were performed for all participants who had taken part in baseline and post-assessment (Model I-Completers). The model included one withinsubject factor (change from pre- to post-assessment) and one between-subject factor (IG vs CG). A significant interaction 


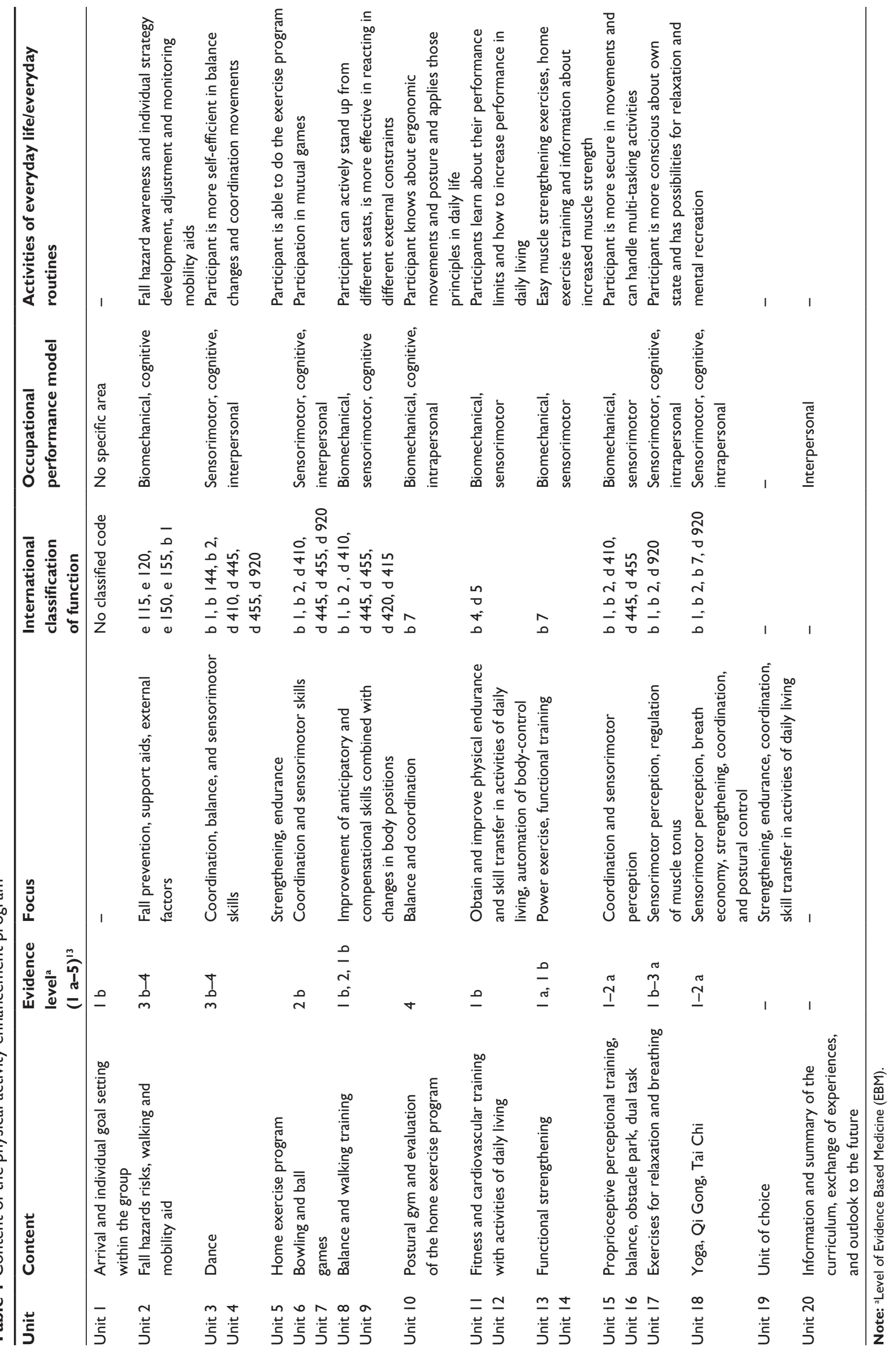


(change $\times$ group) is interpreted as a treatment effect. Effect sizes are provided in terms of partial eta ${ }^{2}$. According to Kirk values of $0.01,0.06$, and 0.14 represent a small, medium, and large effect, respectively. ${ }^{24}$

In case of a significant interaction, further secondary analyses were performed in order to obtain a more precise picture of the treatment effect:

1) Student's $t$-test for paired samples were performed to analyze group differences for the pre- and post-assessments for completers (Model I-Completers).

2) A treatment analysis (Model II-Treatment) was performed to examine effect sizes for those who actually took part in the intervention. Therefore, a GLMMRM was performed excluding those members of the IG who took part in assessments but never took part in any intervention unit.

3) In a third step (Model III-Treatment Adherence) we included the frequency of participation in intervention sessions in the GLMMRM in order to analyze if participants with high participation rates ( $\geq 50 \%)$ benefited more from the intervention than participants with low participation rates $(<50 \%)$. Again those members of the IG who took part in assessments but never took part in any intervention unit were excluded.

4) Finally an intention-to-treat (ITT) analysis (Model IVITT) was performed, which is a conservative calculation method including dropouts. An ITT aims at estimating the overall effect of an intervention by including all initially randomized participants. For dropouts, missing values at post intervention were replaced by the values obtained at baseline levels.

Due to the small number of males, an analysis of sex differences was impossible. However, sex and age were included as covariates in all analyses. The level of significance for all analyses was set at $\alpha=0.05$. Post hoc power analysis for the main results (subjective health status) revealed an achieved power of 0.92 for the obtained interaction effect.

\section{Results \\ Description of sample}

Out of the 276 residents initially involved, 222 (80.4\%) completed the study (nearly equally distributed among the three participating RAC units). At the end-point the IG consisted of 104 participants; the CG comprised 118 participants. Reasons for quitting the program were death, acute disease or prolonged hospital stays, but also refusal to do a second interview.

The average age in the IG was 84 years (minimum: 65 years; maximum: 100 years); in the $C G$ it was rather similar with an average of 85 years ranging from 72 to 99 years; $88 \%$ of the IG were female ( $86 \%$ in CG). Both groups adequately represent the age and sex composition of the residents within the provider organization. There were no significant differences concerning age, sex, and form of living (apartment vs nursing ward and intermediate care) between both groups. The majority of the participants still lived in apartments with household support; a minority lived in intermediate care or on nursing wards (Table 2).

\section{Participation in exercise units}

Twenty exercise units were carried out in a weekly interval with an average of nine participants per group. Members of the IG attended on average 11.7 of the 20 units of the program (mean); this means an average participation rate of $58.5 \%$. Starting from a participation rate of $77.7 \%$ at unit 1 , the rate declined to $51.1 \%$ at unit 10 and then stayed relatively stable

Table 2 Sample composition - characteristics of intervention vs control group

\begin{tabular}{|c|c|c|c|}
\hline & Intervention group & Control group & Test statistic \\
\hline Sample size $(\mathrm{N})$ & 104 & 118 & \\
\hline $\operatorname{Sex}(N)$ & & & Chi-square $=0.055 ; P=0.815$ \\
\hline Females & 91 (87.5\%) & $102(86.4 \%)$ & \\
\hline Males & $13(12.5 \%)$ & $16(13.6 \%)$ & \\
\hline Mean age (standard deviation) & $83.92(6.54)$ & $85.32(5.11)$ & $t=1.787 ; P=0.075$ \\
\hline Form of living $(\mathrm{N})$ & & & Chi-square $=4.078 ; P=0.130$ \\
\hline Apartment & 91 (87.5\%) & II 2 (94.9\%) & \\
\hline Apartment with (temporary) nursing assistance & $5(4.8 \%)$ & $3(2.5 \%)$ & \\
\hline Nursing ward & $8(7.7 \%)$ & $3(2.5 \%)$ & \\
\hline Provider organization $(\mathrm{N})$ & & & Chi-square $=3.556 ; P=0.169$ \\
\hline Organization A & $25(24.0 \%)$ & $42(35.6 \%)$ & \\
\hline Organization B & $42(40.4 \%)$ & $39(33.1 \%)$ & \\
\hline Organization C & $37(35.6 \%)$ & $37(31.4 \%)$ & \\
\hline
\end{tabular}


(endpoint unit 20: 42.5\%). Nearly two thirds of participants $(64.4 \%)$ attended more than ten units; $36.5 \%$ participated in most units (16 to 20). Out of the designated members of the IG who participated in the first and second assessment, $5.8 \%$ never attended the training - as argued above, these were excluded from the treatment analysis.

\section{Effectiveness of the intervention}

Results regarding the effectiveness of the intervention are presented in Table 3. The intervention proved to be effective on subjective health status, as measured by a visual rating scale (EQ-5D) ranging from 0 to 100 . A significant intervention effect was found $\left(P=0.001, \eta_{p}^{2}=0.047\right)$. Selfrated health in the IG increased equaling a small but significant effect ( $P=0.001, d=0.356)$, whereas the $\mathrm{CG}$ showed a slightly negative and not significant change. Further analyses helped to obtain a more precise picture of the treatment effect. As anticipated, the intervention effect increased when excluding members of the IG who actually received no intervention (Model II-Treatment) $\left(P=0.001, \eta_{p}{ }^{2}=0.049\right)$ and further accounting for frequency of participation (Model III-Treatment Adherence) $\left(P=0.004, \eta_{p}^{2}=0.053\right)$. Comparing means of groups according to frequency of participation (Model III-Treatment Adherence) indicates that those participating more often benefitted the most; a result which is plausible. The significant treatment effect concerning subjective health status was also confirmed when using the conservative ITT analysis (Model IV), which accounts for dropout effects $\left(P=0.003, \eta_{p}^{2}=0.033\right)$.

Regarding other health outcomes like health-related QoL (EQ-5D-Index), occupational performance (COPM), as well as pain (visual analog scale), cognitive (MMSE) and objective functional outcome measures (eg, TUAG, CSAR, BS) no significant treatment effects could be observed. Neither the Lower Back Scratch Test nor the Neck Reach Test showed notable effects.

Yet, regarding subjective health measures like healthrelated QoL (EQ-5D-Index) and occupational performance (COPM) a positive but non-significant trend was observed.

As to EQ-5D index (ranging from 0 to 1 ), there is a tendency of stabilization within the IG (pre: 0.77 , post: 0.77 ), whereas the $\mathrm{CG}$ has to deal with a slight decline of healthrelated QoL (pre: 0.75, post: 0.71). COPM performance domain (ranging from 0 to 10 ) measuring performance of everyday life tasks perceived as problematic showed a slight improvement in the IG (pre: 5.18, post: 5.49), while scores in the CG stayed rather stable (pre: 5.24, post: 5.33).

\section{Unexpected adverse effects}

During the study trial, no unexpected adverse events or harm - eg, falls during exercise units or afterward due to less careful behavior - were reported. Further, results of the pain screening indicate no adverse treatment effect $(P=0.85)$; there was a slight non-significant decrease in reported pain in both groups. These findings indicate that the design of the intervention and resources of trainers (selection, training, and support by supervisors in more complex exercise units) were adequate to minimize the risks inherent in PA for this vulnerable population.

\section{Discussion Effectiveness of a low-threshold PA intervention in RAC}

Residents of aged-care facilities are a heterogeneous group in terms of health status, with a high risk for functional decline and loss of independence. As institutions take over many of their everyday activities and duties, a pro-active enhancement of mobility as a basis for autonomy is important in balancing this loss of activities in everyday life. Nevertheless, PA programs often seem designed for younger elderly who suffer little functional impairments. As a result, the program tested in this study was designed to target users of RAC in a representative way by including individuals with no or little impairment, persons with mild to moderate physical and cognitive impairments as well as elderly with low motivation.

Targeting a broad variety of functional dimensions and low frequency of conduct (ie, weekly training) were decisions which - according to the literature - might have had critical implications for the effectiveness of the program on certain dimensions. Nevertheless, the PA program proved to be effective in improving participants' subjective health status; this finding has been supported also by a follow-up study which shows that this effect has remained stable over a period of 1 year after the end of the intervention. ${ }^{25}$

In detail, the results of the present study showed a positive effect on health-related QoL as evidenced by a significant group $\times$ time interaction effect. At post-assessment, the IG showed a significant improvement of QoL whereas there was no significant change in the CG. Small between-group differences at baseline can be judged as coincidence due to randomization. Floor or ceiling effects that cause some kind of a regression to the median are also very unlikely because mean QoL values are located in the middle area of the scale ranging from 0 to 100 . 
Table 3 Results of effectiveness of the intervention on health-related quality of life and other health outcomes

\begin{tabular}{|c|c|c|c|c|}
\hline & Baseline (SD) & Post-assessment (SD) & $P$-value & Partial eta $^{2}$ \\
\hline \multicolumn{5}{|l|}{ Main result } \\
\hline \multicolumn{5}{|c|}{ Subjective health status (EQ-5D visual analog scale) } \\
\hline \multicolumn{5}{|l|}{ GLMMRM $^{\mathrm{a}}$} \\
\hline \multicolumn{5}{|l|}{ Model I-Completers } \\
\hline Intervention $(n=102)$ & $54.29(24.54)$ & $62.01(17.27)$ & $0.001 * *$ & 0.047 \\
\hline Control $(n=114)$ & $64.87(18.86)$ & $62.63(18.29)$ & & \\
\hline \multicolumn{5}{|l|}{ Model II-Treatment } \\
\hline Intervention $(n=96)$ & $54.67(24.56)$ & $62.55(17.62)$ & $0.001 * *$ & 0.049 \\
\hline Control $(n=114)$ & $64.87(18.86)$ & $62.63(18.29)$ & & \\
\hline \multicolumn{5}{|l|}{ Model III-Treatment Adherence } \\
\hline Intervention ( $\leq 10$ units) $(n=30)$ & $54.93(24.97)$ & $58.67(17.56)$ & $0.004 * *$ & 0.053 \\
\hline Intervention $(>10$ units) $(n=66)$ & $54.55(24.56)$ & $64.32(17.50)$ & & \\
\hline Control $(n=114)$ & $64.87(18.86)$ & $62.63(18.29)$ & & \\
\hline \multicolumn{5}{|l|}{ Model IV-ITT } \\
\hline Intervention $(n=139)$ & $56.67(24.21)$ & $62.33(18.66)$ & $0.003 * *$ & 0.033 \\
\hline Control $(n=135)$ & $64.81(18.16)$ & $62.93(17.68)$ & & \\
\hline Student's $t$-test for paired samples & Paired mean difference (SD) & & $P$-value & Cohen's d \\
\hline \multicolumn{5}{|l|}{ Model I-Completers } \\
\hline Intervention $(n=102)$ & $7.72(23.27)$ & & $0.001 * *$ & 0.356 \\
\hline Control $(n=1 \mid 4)$ & $-2.24(20.56)$ & & 0.248 & 0.120 \\
\hline \multicolumn{5}{|l|}{ Further selected results } \\
\hline Outcome & Baseline (SD) & Post-assessment (SD) & $P$-value & Partial eta ${ }^{2}$ \\
\hline \multicolumn{5}{|c|}{ Health-related quality of life (EQ-5D-Index) and occupational performance (COPM-performance) } \\
\hline \multicolumn{5}{|l|}{ GLMMRMa Model I } \\
\hline \multicolumn{5}{|l|}{ EQ-5D-Index } \\
\hline Intervention ( $\mathrm{n}=99)$ & $0.77(0.24)$ & $0.77(0.23)$ & 0.158 & 0.010 \\
\hline Control $(n=11 I)$ & $0.75(0.25)$ & $0.71(0.28)$ & & \\
\hline \multicolumn{5}{|l|}{ COPM-performance } \\
\hline Intervention $(n=85)$ & $5.18(1.84)$ & $5.49(2.01)$ & 0.292 & 0.006 \\
\hline Control ( $n=97)$ & $5.24(1.91)$ & $5.33(2.14)$ & & \\
\hline \multicolumn{5}{|c|}{ Pain (visual analog scale) and cognitive functioning (MMSE) } \\
\hline \multicolumn{5}{|l|}{ GLMMRMa Model I } \\
\hline \multicolumn{5}{|l|}{ Pain } \\
\hline Intervention $(n=104)$ & $3.68(2.32)$ & $3.46(2.43)$ & 0.850 & $<0.001$ \\
\hline Control $(n=117)$ & $3.60(2.60)$ & $3.43(2.75)$ & & \\
\hline \multicolumn{5}{|l|}{ MMSE (shortened form) } \\
\hline Intervention $(n=104)$ & $14.15(1.73)$ & $14.08(2.19)$ & 0.508 & $<0.002$ \\
\hline Control $(n=114)$ & I4.II (2.18) & $14.08(2.48)$ & & \\
\hline \multicolumn{5}{|c|}{ Objective functional health outcomes (Timed Up and Go/Chair Sit and Reach/Back Scratch Test) } \\
\hline \multicolumn{5}{|l|}{ GLMMRMa Model I } \\
\hline \multicolumn{5}{|l|}{ Timed $U_{p}$ and Go Test ${ }^{\mathrm{b}}$} \\
\hline Intervention $(n=74)$ & $16.69(13.30)$ & $17.89(\mid 1.64)$ & 0.240 & 0.009 \\
\hline Control $(n=85)$ & $18.19(10.70)$ & $18.14(10.70)$ & & \\
\hline \multicolumn{5}{|c|}{ Chair Sit and Reach Test (Average R+L leg) } \\
\hline Intervention $(n=93)$ & $-12.15(12.28)$ & $-14.45(13.72)$ & 0.089 & 0.016 \\
\hline Control $(n=94)$ & $-12.28(12.07)$ & $-11.94(13.00)$ & & \\
\hline \multicolumn{5}{|l|}{ Back Scratch Test (Average R+L arm) } \\
\hline Intervention $(\mathrm{n}=7 \mathrm{I})$ & $-20.75(|2.0|)$ & $-20.62(|0.7|)$ & 0.338 & 0.007 \\
\hline Control $(n=72)$ & $-19.06(11.52)$ & $-19.91(12.09)$ & & \\
\hline
\end{tabular}

Notes: $* * P<0.01$. a GLMMRM, generalized linear mixed models for repeated measures (reported $P$-values and partial eta ${ }^{2}$ refer to the interaction effect [change $\times$ groups] which reflects the treatment effect) controlled for sex and age; bonly cases included who used either no or the same walking aid at pre- and post-assessment.

Abbreviations: ITT, intention-to-treat; COPM, Canadian Occupational Performance Measure; MMSE, Mini-Mental State Examination (shortened form; range: 0-I5); $\mathrm{R}$, right; L, left; SD, standard deviation; EQ-5D, Euro Quality of Life-5 dimensions. 
Even though we found only a small intervention effect on residents' subjective health status, this result seems important in the context of RAC. First, studies have shown associations between subjective health status and mortality which persists after adjustments for other health indicators and socio-demographic covariates. ${ }^{26}$ Second, health-related QoL is of central importance in the context of LTC as it is seen as one of the most relevant and appropriate constructs to address quality of care and health of residents in LTC. The construct comprises aspects of well-being that are affected by progressive health status changes as well as by the health care provided to address these complex and changing needs. The focus on individuals' rather than health professionals' opinions emphasizes the principle of "patient centered care". ${ }^{27}$ The lack of notable effects concerning selected functional outcome measures is coherent, seen in the context of this adjusted, low intensity program design. And last but not least in the context of a general trend toward decreasing health status in users of RAC, even small improvements or stabilization of health can be considered as important results.

\section{Implications for the design of low- threshold PA interventions in RAC}

The aim of this study is to prove that a low-threshold PA intervention in RAC can be effective in promoting residents' health. Qualitative feedback from trainers and staff helped to detect critical elements in the design of this kind of intervention and give guidance for future studies in this area.

First, pro-active recruitment and ongoing active support for participation seems relevant to reach a larger group of residents, especially those with impairment or little personal motivation for PA. This was achieved by personal invitation (ie, face-to-face contact) creating the opportunity to discuss pros and cons. Moderate to bad health status had been previously identified as a barrier concerning PA in the aged, ${ }^{9}$ so emphasizing health benefits for this group seemed relevant. There are some indications that inactive residents who had not taken part in exercise groups beforehand, were motivated through pro-active recruitment. Consistent with this finding, the literature suggests that social support like encouragement (eg, from the family or a physician) can be a critical motive for older adults to start exercising. ${ }^{9,28}$ Social interaction and the group setting have also been shown to be major motivation factors for participating in PA programs for the aged., ${ }^{9,29}$
Second, the principle of individualization seemed to be very important in increasing retention. Individualization was supported by creating groups according to functional capacities. Tailoring exercises to meet the needs of individuals and providing home training programs further promoted an individualized intervention. Individualization may have counteracted another barrier: in addition to poor health acting as a hurdle between inactive elderly and exercise, ${ }^{30}$ Costello et al had found that intimidation can be an additional barrier for inactive elderly to join an established activity program; there are fears of slowing down the class and not being able to keep up. ${ }^{31}$ Taking health concerns and intimidation into account, individualization can help to tailor PA and exercises to meet individual capacities and needs. ${ }^{31,32}$

Yet with regard to cognitively impaired aged persons, the low turnout of this group and some critical feedback from the trainers in focus groups indicated that it is difficult to integrate them in such a group program; at this stage, more individualized therapeutic interventions might be needed.

\section{Study limitations}

The study design implies some limitations concerning the generalizability of findings for RAC. The group of aged with severe physical or cognitive impairments was excluded from participation - therefore the results are limited to the group of residents with no-to-moderate physical, and no-tominor cognitive impairment. Another limitation is the lack of information about the reasons given by a large number of residents who declined to participate in the study. To be able to distinguish motivational issues from other considerations would be very valuable for creating still more inclusive programs. Further, the eligibility decision was based on the judgment of the local coordinators, who were able to base the eligibility decision upon some knowledge of the residents' health status. A more systematic monitoring of the recruitment process and applying objective measures as a basis for the eligibility decision would have supported objective traceability of the recruitment process.

Concerning negative events like falls, nothing was reported, but these events were also not defined as outcomes in advance and thus not systematically monitored.

Finally, as the participants' medical status and history, especially the quality of their pain management, were not documented, it could not be included in the data analyses. Inclusion of these measures in future studies is recommended. 


\section{Need for research}

Therapeutic interventions for frail elderly patients have been described in recent publications, ${ }^{33,34}$ but there seems to be a need for future research to further explore, deepen, and confirm findings about the effectiveness especially of low-threshold PA interventions in RAC. Further, there seems too little research on underlying mechanisms that enable and enhance residents' participation in exercise programs including more intense programs. We can see a strong need for studies to shed light on these complex dynamics in order to develop and investigate supportive measures. The present study provides implications that pro-active support and individualization might be important measures.

\section{Conclusion}

The study demonstrates that it is possible to design and implement a complex, evidence-based PA intervention for residents with no or mild physical and/or cognitive impairment and recruit and retain a relatively large part of the residents in this program. One important reason for this success was that limited organizational and residents' resources were taken into account. The study proved effectiveness of this PA intervention in improving residents' subjective health status. Pro-active recruitment and organized social support appear to be effective measures to counteract self-selection, explicitly also including formerly inactive residents. In carrying out the intervention, several measures appear to be feasible and relevant for motivating participants.

\section{Acknowledgments}

We would like to thank Austrian Health Promotion Foundation (Rainer Christ), Main Association of Austrian Social Security Organizations (Sascha Müller), Vienna Health Promotion Inc. (Ursula Hübel), and our practice partner KWP (Eva Bader, Thomas Krupinski, Erika Mosor, Ehrentraud Svoboda, and Eva Solar-Neubauer) for their support to develop and implement the PA intervention. Financial support for the research and/or publication of this article was provided by the Ludwig Boltzmann Society, a private nonprofit association organizing research, funded by the Austrian Federal Ministry for Science and Research.

\section{Disclosure}

The authors declare no potential conflicts of interest with respect to the research, authorship, and/or publication of this article.

\section{References}

1. Horn A, Brause M, Schäffer D. Bewegungsförderung in der (stationären) Langzeitversorgung [Enhancement of physical activity in (residential) long-term care]. In: Geuter G, Hollederer A, editors. Handbuch Bewegungsförderung und Gesundheit. Bern: Hans Huber; 2012:305-318. German.

2. Chin A Paw MJ, van Uffelen JG, Riphagen I, van Mechelen W. The functional effects of physical exercise training in frail older people: a systematic review. Sports Med. 2008;38(9):781-793.

3. Stathi A, Simey P. Quality of life in the Fourth Age: exercise experiences of nursing home residents. J Aging Phys Act. 2007;15(3):272-286.

4. Weening-Dijksterhuis E, de Greef MH, Scherder EJ, Slaets JP, van der Schans CP. Frail institutionalized older persons: A comprehensive review on physical exercise, physical fitness, activities of daily living, and quality-of-life. Am J Phys Med Rehabil. 2011;90(2):156-168.

5. Forster A, Lambley R, Young JB. Is physical rehabilitation for older people in long-term care effective? Findings from a systematic review. Age Ageing. 2010;39(2):169-175.

6. Chin A Paw MJ, van Poppel MN, Twisk JW, van Mechelen W. Once a week not enough, twice a week not feasible? A randomised controlled exercise trial in long-term care facilities [ISRCTN87177281]. Patient Educ Couns. 2006;63(1-2):205-214.

7. Rydwik E, Frandin K, Akner G. Physical training in institutionalized elderly people with multiple diagnoses - a controlled pilot study. Arch Gerontol Geriatr. 2005;40(1):29-44.

8. Lazowski DA, Ecclestone NA, Myers AM, et al. A randomized outcome evaluation of group exercise programs in long-term care institutions. J Gerontol A Biol Sci Med Sci. 1999;54(12):M621-M628.

9. Baert V, Gorus E, Mets T, Geerts C, Bautmans I. Motivators and barriers for physical activity in the oldest old: a systematic review. Ageing Res Rev. 2011;10(4):464-474.

10. Ferrucci L. The Baltimore Longitudinal Study of Aging (BLSA): a 50-year-long journey and plans for the future. J Gerontol A Biol Sci Med Sci. 2008;63(12):1416-1419.

11. Krajic K, Cichocki M, Quehenberger V. Health-promoting residential aged care: a pilot project in Austria. Health Promot Int. Epub 2014 Mar 28.

12. Stamm T, Diermayr G. Curriculum Für die interdisziplinäre Intervention „Bewegtes Leben “im Projekt „, Gesundheit hat kein Alter [Curriculum for the interdisciplinary intervention "Moving Life" as part of the project "Health has no age"]; 2011. Available from: http://www. gesundheithatkeinalter.at/Publikationen/Publikationen/Curriculumfuer-die-interdisziplinaere-Intervention-Bewegtes-Leben-im-ProjektGesundheit-hat-kein-Alter. Accessed March 25, 2015. German.

13. CEBM.net [homepage on the Internet]. University of Oxford: Centre for Evidence Based Medicine; Levels of Evidence; 2009). Available from: http://www.cebm.net/index.aspx?o=1025. Accessed December 12, 2014.

14. EuroQol Group. EuroQol - a new facility for the measurement of health-related quality of life. Health Policy. 1990;16(3):199-208.

15. Scott J, Huskisson EC. Graphic representation of pain. Pain. 1976; 2(2):175-184.

16. Law M, Baptiste S, Carswell A, et al. Canadian Occupational Performance Measure: Vierte Auflage. Lizenzierte deutsche Ausgabe. dt. Übersetzung: Dehnhardt B, George S, Harth A. Idstein: SchulzKirchner; 2009.

17. Bohannon RW. Reference Values for the Timed Up and Go Test. A Descriptive Meta-Analysis. J Geriatr Phys Ther. 2006;29(2):64-68.

18. Podsiadlo D, Richardson S. The Timed "Up and Go": a test of basic functional mobility for frail elderly persons. J Am Geriatr Soc. 1991; 39(2):142-148.

19. Rydwik E, Bergland A, Forsen L, et al. Psychometric Properties of Timed Up and Go in Elderly People. A Systematic Review. Phys Occup Ther Geriatr. 2011;29(2):102-125. 
20. Jones CJ, Rikli RE. Measuring functional fitness of older adults. The Journal on Active Aging. 2002:24-30.

21. Niethard FU, Pfeil J, Biberthaler P. Orthopädie und Unfallchirurgie [Orthopedics and Traumatology]. Stuttgart: Georg Thieme Verlag KG; 2009. German.

22. Haubois G, Annweiler C, Launay C, et al. Derivation and validation of a Short Form of the Mini-Mental State Examination for the screening of dementia in older adults with a memory complaint. Eur J Neurol. 2013;20(3):588-590.

23. Gusi N, Prieto J, Olivares PR, et al. Normative fitness performance scores of community-dwelling older adults in Spain. J Aging Phys Act. 2012;20(1):106-126.

24. Kirk RE. Practical significance: A concept whose time has come. Educ Psychol Meas. 1996;56(5):746-759.

25. Quehenberger V, Cichocki M, Krajic K. Sustainable effects of a lowthreshold mobility enhancement intervention on health-related quality of life in residential aged care. Clin Interv Aging. 2014;9:1853-1864.

26. Idler EL, Benyamini Y. Self-rated health and mortality: a review of twentyseven community studies. J Health Soc Behav. 1997;38(1):21-37.

27. Zubritsky C, Abbott KM, Hirschman KB, et al. Health-related quality of life: expanding a conceptual framework to include older adults who receive long-term services and supports. Gerontologist. 2013;53(2): 205-210.
28. Grossman MD, Stewart AL. "You aren't going to get better by just sitting around": physical activity perceptions, motivations, and barriers in adults 75 years of age or older. Am J Geriatr Cardiol. 2003; 12(1):33-37.

29. Brenner I, Marsella A. Factors influencing exercise participation by clients in long-term care. Perspectives. 2008-2009;32(4):5-11.

30. Lim K, Taylor L. Factors associated with physical activity among older people - a population-based study. Prev Med. 2005;40(1):33-40.

31. Costello E, Kafchinski M, Vrazel J, Sullivan P. Motivators, barriers, and beliefs regarding physical activity in an older adult population. J Geriatr Phys Ther. 2011;34(3):138-147.

32. Boehm J, Franklin RC, Newitt R, et al. Barriers and motivators to exercise for older adults: A focus on those living in rural and remote areas of Australia. Aust J Rural Health. 2013;21(3):141-149.

33. Bibas L, Levi M, Bendayan M, et al. Therapeutic interventions for frail elderly patients: part I. Published randomized trials. Prog Cardiovasc Dis. 2014;57(2):134-143.

34. Bendayan M, Bibas L, Levi M, et al. Therapeutic interventions for frail elderly patients: part II. Ongoing and unpublished randomized trials Prog Cardiovasc Dis. 2014;57(2):144-151.
Clinical Interventions in Aging

\section{Publish your work in this journal}

Clinical Interventions in Aging is an international, peer-reviewed journal focusing on evidence-based reports on the value or lack thereof of treatments intended to prevent or delay the onset of maladaptive correlates of aging in human beings. This journal is indexed on PubMed Central, MedLine,

\section{Dovepress}

CAS, Scopus and the Elsevier Bibliographic databases. The manuscript management system is completely online and includes a very quick and fair peer-review system, which is all easy to use. Visit http://www.dovepress. com/testimonials.php to read real quotes from published authors. 